\title{
La drosophile: un modèle pour l'étude de la réponse immunitaire innée
}

\section{Bruno Lemaitre}

ADRESSE

B. Lemaitre: chargé de recherche au Cnrs. Centre de génétique moléculaire, Cnrs, 91198 Gif-sur-Yvette, France.

TIRÉS À PART

B. Lemaitre.

Les insectes possèdent des mécanismes de défense contre les infections très efficaces - fondés sur des réactions cellulaires et humorales - qui ont vraisemblablement contribué à leur succès évolutif. Les réactions cellulaires impliquent les hémocytes qui participent à l'encapsulement ou à la phagocytose des particules étrangères. Le volet humoral consiste, d'une part, dans l'activation de cascades protéolytiques (coagulation et mélanisation) et, d'autre part, dans la synthèse de peptides antimicrobiens. Plusieurs voies de régulation gouvernent l'expression des gènes codant pour les peptides antimicrobiens; l'une d'elles, la voie Toll, présente de fortes similitudes structurales et fonctionnelles avec une voie de transmission du signal qui, chez les mammifères, gouverne l'expression des gènes de la réponse immunitaire. Cette voie de transmission du signal est donc une cascade évolutivement très ancienne impliquée dans les mécanismes de défense des eucaryotes; des similitudes frappantes existent entre la réponse antimicrobienne de l'insecte et la réponse innée non adaptative des mammifères.

A u cours de l'évolution, deux systèmes immunitaires de lutte contre les agents infectieux ont été sélectionnés: l'immunité innée et l'immunité adaptative [1]. L'immunité adaptative a été la plus étudiée. Elle présente deux caractéristiques essentielles: la spécificité, fondée sur l'existence d'un large répertoire de molécules de reconnaissance qui sont engendrées par des réarrangements somatiques (immunoglobulines, récepteurs des cellules T) et la mémoire, grâce à la sélection clonale de lymphocytes spécifiques. Cette réponse est apparue il y a environ 400 millions d'années et n'existe que chez les vertébrés. L'immunité innée est phylogénétiquement plus ancienne et existe vraisemblablement chez tous les métazoaires. Chez les mammifères, cette réponse constitue la première ligne de défense contre les infections. La réponse immunitaire innée ne fait pas appel à des mécanismes de reconnaissance impliquant des immunoglobulines. Elle assurerait une discrimination entre le soi et le non-soi infectieux grâce à des récepteurs capables de reconnaître des molécules aux motifs structuraux invariants présents sur l'enveloppe des micro-organismes mais absents des cellules eucaryotes (exemple: récepteur des lipopolysaccharides (LPS), récepteur du mannose). Lorsque ces récepteurs sont stimulés, ils déclenchent immédiatement des mécanismes effecteurs semblables à 
ceux activés par l'immunité adaptative (phagocytose, opsonisation, cascade du complément...). Malgré son succès du point de vue évolutif, l'immunité innée n'a été que peu étudiée par les immunologistes. Cependant, depuis peu, de nouvelles données ont permis de reconsidérer son rôle. Cette réponse participerait non seulement à l'activation de mécanismes de défense immédiats mais serait de plus une étape obligatoire déterminant la nature de la réponse adaptative mise en place par la suite [2].

Les insectes représentent plus de $90 \%$ des espèces animales. Ils ont colonisé toutes les niches écologiques terrestres et sont de ce fait confrontés à une extrême variété de micro-organismes. Les insectes possèdent des mécanismes de défense très efficaces contre les infections qui ont vraisemblablement contribué à leur succès évolutif $[3,4]$. Ils sont fondés sur des réactions cellulaires et humorales. Les premières impliquent les hémocytes qui participent à l'encapsulation ou à la phagocytose des particules étrangères. Le volet humoral consiste, d'une part, en l'activation de cascades protéolytiques (coagulation et mélanisation) et, d'autre part, en la synthèse de peptides antimicrobiens.

Jusqu'au début des années 1990, il était largement admis que les réactions immunitaires des insectes relevaient de mécanismes totalement différents de ceux des vertébrés. Des études moléculaires et génétiques récentes, réalisées chez la drosophile, révèlent aujourd'hui l'existence de similitudes frappantes entre la réponse innée des mammifères et la défense antimicrobienne des insectes.

\section{La réponse humorale antimicrobienne}

L'injection de bactéries ou même une simple blessure induit chez les insectes les plus évolués la synthèse d'une batterie de peptides antimicrobiens. Ces molécules antibiotiques sont produites par le corps gras, un organe fonctionnellement analogue du foie des vertébrés, et sont sécrétées dans l'hémolymphe [3, 4]. Chez la drosophile, plus de sept peptides antimicrobiens ont été caractérisés. Cinq d'entre eux (diptéricine, cécropine, attacine, drosocine, défensine) contre les bactéries. La drosomycine est active contre les champignons. Enfin, la metchnikowine possède une activité à la fois antibactérienne et antifongique. Ces molécules présentent des motifs structuraux qui sont conservés dans des peptides antimicrobiens isolés dans d'autres phylums. Leur mode d'action est mal connu mais on pense que ces petits peptides cationiques se fixent au niveau des membranes des microorganismes et les tuent en les perméabilisant (voir l'article de Philippe Bulet, p. 23 de ce numéro).

Les gènes codant pour ces peptides ont été clonés chez la drosophile. Il s'agit généralement de petits gènes dépourvus d'introns qui sont dispersés à diverses positions dans le génome. Les quatre gènes codant pour les cécropines échappent à cette règle puisqu'ils sont situés dans un complexe génique et possèdent un intron. Tous ces gènes codent pour des protéines comportant une séquence signal qui permet leur sécrétion dans l'hémolymphe. Ils sont peu (ou pas) exprimés en condition normale. L'injection de bactéries induit rapidement une très forte expression de ces gènes. Cette expression débute moins de 60 minutes après infection, atteint son maximum après 6-12 heures, puis décline lentement. Les peptides produits vont s'accumuler dans l'hémolymphe et atteindre des concentrations élevées (jusqu'à $100 \mu \mathrm{M}$ pour la drosomycine) permettant la destruction des germes infectieux $[3,4]$.

La réponse humorale antimicrobienne est contrôlée essentiellement au niveau transcriptionnel. Une telle régulation peut être aisément visualisée chez des drosophiles portant un transgène avec une fusion entre le promoteur d'un gène codant pour un de ces peptides et un gène "rapporteur». Récemment, des constructions utilisant comme rapporteur le gène de la GFP (green fluorescent protein) ont permis d'étudier le profil d'expression spatio-temporel de ces gènes en réponse à une infection chez des drosophiles vivantes [5]. Le corps gras apparaît alors comme le principal tissu dans lequel sont exprimés les gènes antimicrobiens (figure 1). Cette étude a aussi montré que les gènes codant pour les peptides antimicrobiens sont exprimés dans de nom- breux épithéliums en relation avec le milieu extérieur, comme par exemple les trachées. La réponse locale joue certainement un rôle dans la lutte contre les infections dans ces tissus. En éliminant l'agent pathogène sur le site d'infection, elle permet d'éviter l'activation d'une réponse systémique fort coûteuse d'un point de vue énergétique. Cette réponse locale est à mettre en parallèle avec la situation observée chez les mammifères chez lesquels les peptides antimicrobiens jouent un rôle fondamental de défense dans les muqueuses [6].

\section{Les protéines Rel de la drosophile}

Les promoteurs de plusieurs gènes codant pour des peptides antimicrobiens ont été analysés en détail. Ils présentent des motifs homologues de séquences cis-régulatrices présentes dans les promoteurs des gènes induits au cours de la réponse immunitaire des mammifères [7-9]. Il s'agit essentiellement d'éléments de réponse à l'interleukine-6 (IL-6 RE), et à l'interféron, et des motifs GATA et $\mathrm{kB}$ (figure 2). Divers travaux ont démontré que les motifs $\kappa B$ étaient nécessaires pour l'inductibilité des gènes antibactériens après blessure septique $[10,11]$.

Chez les mammifères, les motifs $\kappa B$ sont le site de fixation de $\mathrm{NF}-\kappa \mathrm{B}$, un facteur de transcription inductible qui joue un rôle important dans le contrôle des gènes induits au cours de la réponse immunitaire et inflam-

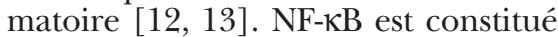
d'un hétérodimère de deux protéines de la famille Rel (relA et p50). Les protéines Rel sont caractérisées par un domaine commun (domaine Rel) situé dans leur partie amino-terminale, responsable des propriétés de liaison à l'ADN, de dimérisation et de la localisation nucléaire de la protéine. En l'absence de signal, les complexes NF-кB sont séquestrés dans le cytoplasme par une protéine de la famille IкB. Les membres de cette famille se caractérisent par un domaine formé de répétitions ankyrine nécessaires à l'interaction avec NF-кB. En réponse à un signal approprié, I $\kappa \mathrm{B}$ est phosphorylé puis dégradé par le protéasome. La dégradation d'I $\kappa \mathrm{B}$ permet la translocation rapide de $\mathrm{NF}-\mathrm{kB}$ dans le noyau, assurant ainsi l'induction des gènes cibles. 

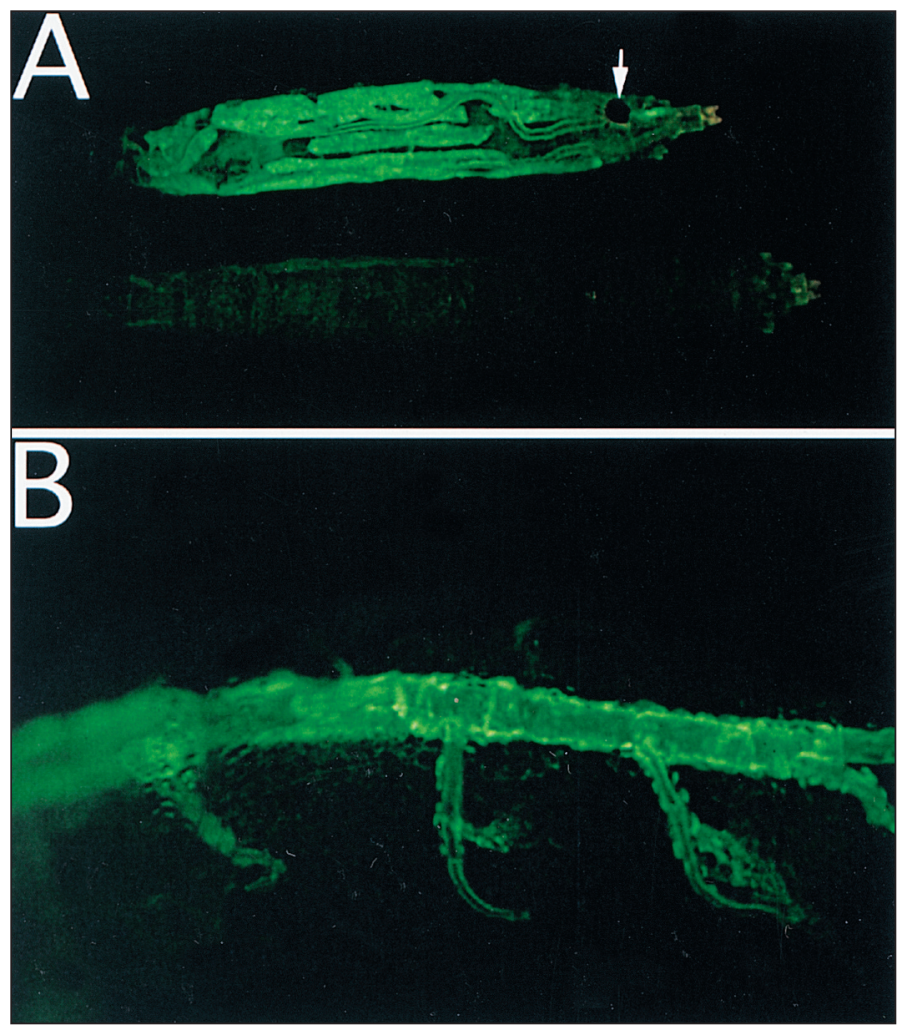

Figure 1. Le rapporteur drosomycine-GFP révèle une réponse immunitaire "localisée " chez la drosophile. A. La réponse humorale systémique. Larve transgénique $24 \mathrm{~h}$ après injection d'un mélange bactérien (haut); larve transgénique en I'absence de blessure (bas); la fluorescence observée correspond à une expression du transgène rapporteur dans le corps gras de la larve et est observée uniquement après infection microbienne. Le transgène utilisé est une fusion entre le promoteur du gène de la drosomycine et le gène rapporteur GFP (green fluorescent protein) qui code pour une protéine fluorescente permettant une observation directe chez des drosophiles vivantes. Notons, chez la larve "immunisée", la présence d'une tache noire (flèche) causée par la réaction de mélanisation au point de blessure. $\boldsymbol{B}$. La réponse localisée chez la larve. Expression du transgène rapporteur dans l'épithélium trachéen de la larve. Cette réponse est limitée à une portion du tronc trachéen et correspond à l'induction d'une réponse antimicrobienne en réponse à une infection dans ce tissu. (D'après [5].)

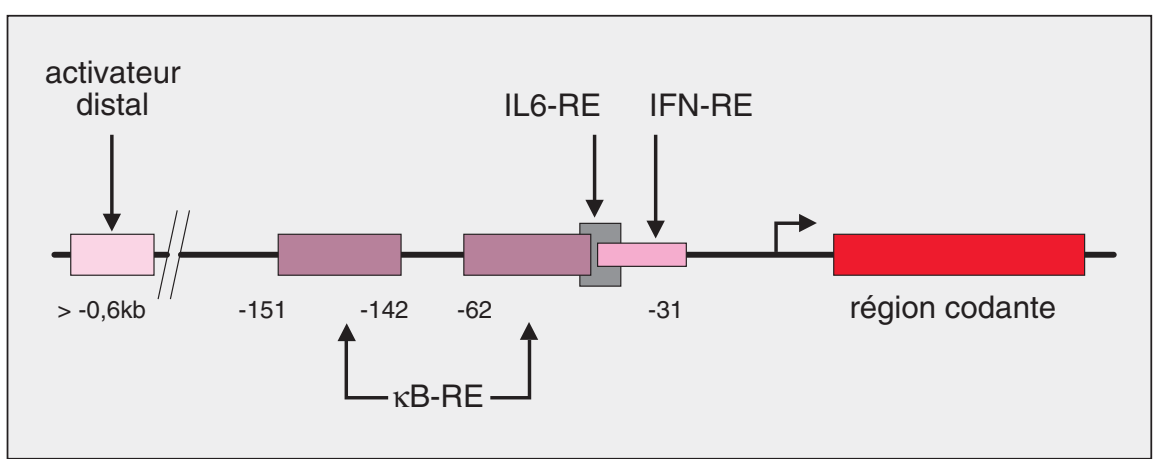

Figure 2. Organisation du promoteur du gène de la diptéricine. Deux éléments de réponse de type $\kappa B$ observés en amont du gène de la diptericine confèrent l'inductibilité à ce gène. D'autres motifs cis-régulateurs sont observés (éléments de réponse à l'interféron et à l'interleukine-6) qui, en association avec une séquence activatrice distale, règlent le niveau d'expression du gène de la diptéricine après infection bactérienne. (D'après [3].)

Ce mécanisme de contrôle qui ne nécessite pas de synthèse protéique préalable est particulièrement adapté au rôle de NF- $\kappa B$ dans l'activation rapide des gènes lors d'une infection

$\mathrm{m} / \mathrm{s} n^{\circ} 1$, vol. 15 , janvier 99 la dégradation $\mathrm{d}^{\prime} \mathrm{I} \kappa \mathrm{B}$, en réponse à un signal extracellulaire, ont pu être décryptées. L'une des voies de transduction du signal les mieux connues est la cascade induite par la fixation de l'interleukine-1 sur son récepteur IL1-R [14]. Cette voie met en jeu des cascades de kinases successives qui vont finalement phosphoryler IкB et induire sa dégradation $(\mathrm{m} / \mathrm{s} 1998$, $n^{\circ} 11, p .1281$ ) (figure 3).

Les protéines Rel ne sont pas limitées aux vertébrés. Trois gènes de drosophile codent pour des protéines qui appartiennent à cette famille (figure 4). Deux d'entre eux, appelés dorsal et dif (dorsal-related immune factor) codent pour des molécules qui présentent une structure identique au prototype RelA: un domaine Rel dans la partie amino-terminale et un domaine transactivateur dans la partie carboxy-terminale $[15,16]$. Ces deux gènes sont situés à moins de 7 kilobases l'un de l'autre sur le chromosome, suggérant qu'ils sont issus d'une duplication. Le troisième est le gène relish, qui, à la différence de dorsal et dif, code pour une protéine présentant une structure semblable à p105, le précurseur de la sous-unité p50 chez la souris [17]. P105 et Relish sont composées d'un domaine de type Rel dans la partie amino-terminale et d'un domaine carboxy-terminal présentant des motifs ankyrine (figure 4). Les données actuelles suggèrent que la partie carboxy-terminale joue un rôle inhibiteur semblable à celui exercé par IкB. L'activation de Relish et de p105 serait précédée d'un clivage protéolytique libérant une protéine Rel dépourvue de domaine inhibiteur.

Plusieurs arguments sont en faveur d'un rôle de ces trois protéines Rel dans le contrôle des gènes codant pour les peptides antimicrobiens chez la drosophile. D'une part, les gènes dorsal, dif et relish sont tous trois exprimés dans le corps gras et leur expression est significativement augmentée après infection microbienne [16-20]. D’autre part, des expériences in vitro indiquent que ces protéines peuvent se fixer sur les motifs $\kappa \mathrm{B}$ présents dans les promoteurs en amont des gènes codant pour les peptides antimicrobiens [18, 20, 21].

Ainsi, en de nombreux points la réponse humorale antimicrobienne de la drosophile évoque la réponse de phase aiguë des mammifères. Cette 


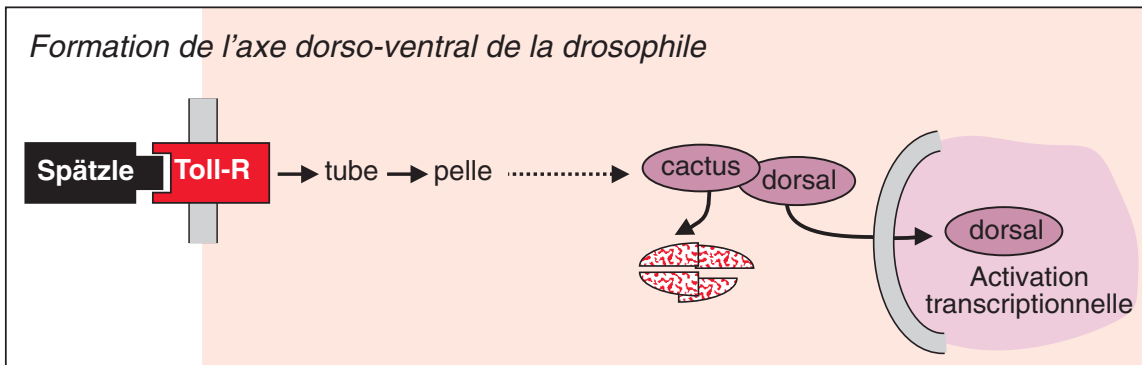

Réponse immunitaire chez les mammifères

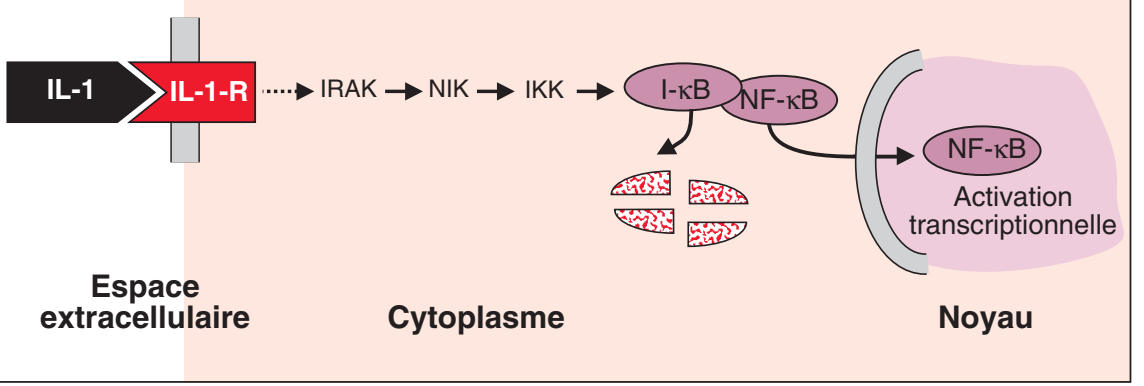

Figure 3. Voies de signalisation conservées menant à la translocation nucléaire de dorsal et NF- $\kappa$ B. Sur la face ventrale de l'embryon de drosophile, la fixation du ligand Spätzle au récepteur Toll induit la transduction du signal par les protéines Tube et Pelle qui aboutit à la dissociation du complexe Dorsal/Cactus et à la translocation de Dorsal dans le noyau. Dans les cellules immunes de mammifères, la liaison de l'interleukine 1 à son récepteur (IL1-R) active une cascade de kinases qui implique IRAK (IL-1 receptorassociated kinase), un homologue de Pelle, NIK (NF- $\kappa \mathrm{B}$ inducible kinase), et le complexe IKK (IKB kinase 1 et 2). Cette activation aboutit à la dissociation

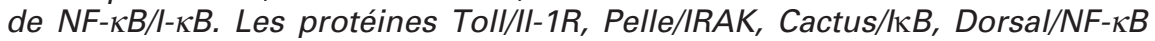
présentent des similitudes de séquence. (D'après [3].)

dernière consiste en la synthèse par le foie d'une série de peptides et polypeptides (exemple: la protéine $\mathrm{C}$ réactive) en réponse à une infection systémique [22]. Le contrôle se fait au niveau de la transcription et implique des transactivateurs de la famille Rel.

\section{La voie Toll et la réponse antifongique}

Dorsal est la protéine Rel de drosophile la mieux connue. Elle joue un rôle dans la mise en place de l'axe dorso-ventral au cours du développement embryonnaire. La translocation nucléaire de Dorsal dans l'embryon est contrôlée par une cascade qui a été bien décryptée [23]. Cette voie de signalisation débute dans le compartiment extracellulaire par l'activation d'une cascade de protéases à sérine qui provoquent la maturation par clivage protéolytique de Spätzle, le ligand du récepteur Toll. L'activation de Toll par son ligand déclenche une voie de signalisation
Tube et la protéine-kinase Pelle et aboutit finalement à la dégradation de Cactus, un inhibiteur qui séquestre la protéine Dorsal dans le cytoplasme. Ce facteur de transcription va alors régler de nombreux gènes cibles responsables de la polarité dorso-ventrale de l'embryon.

La partie intracellulaire de cette cascade, appelée voie Toll, présente de fortes similitudes avec la voie d'activation de NF- $\mathrm{kB}$ par la cytokine IL-1 au cours de la réponse inflammatoire des mammifères (figure 3). Dans les deux cas, la fixation d'un ligand extracellulaire (Spätzle/IL-1) à un récepteur transmembranaire (Toll/IL-1R) induit une cascade de signalisation par l'intermédiaire d'une kinase (Pelle/IRAK), qui aboutit à la dégradation d'une protéine inhibitrice dans le cytoplasme (Cactus/IאB) et à la libération d'une protéine Rel (Dorsal/NF-кB). Celle-ci va induire dans le noyau l'expression de nombreux gènes cibles via les motifs $\kappa B$. Ces parallèles sont particulièrement significatifs si l'on considère que les domaines intracytoplasmiques de Toll et IL-1R ont des structures proches, que les kinases Pelle et IRAK présentent des homologies de séquence, que Cactus et IKB, d'une part, NF- $\kappa \mathrm{B}$ et Dorsal, de l'autre, appartiennent à deux mêmes familles de protéines. Ainsi, la voie Toll/IL-1R est une cascade biochimique conservée entre les mammifères et la drosophile [23].

Une étude récente a montré qu'en plus de sa fonction dans l'embryogenèse précoce, la voie de signalisation Toll (comprenant les produits Spätzle, Toll, Pelle, Tube et Cactus) est réutilisée pour régler l'expression du gène qui code pour la drosomycine au cours de la réponse immunitaire [24]. En effet, l'inductibilité de ce gène est fortement réduite chez des drosophiles portant des mutations dans les gènes spätzle, Toll, pelle, et tube après infection microbienne. Une mutation dans le gène cactus se traduit par une expression constitutive élevée du gène de la drosomycine, suggérant que la protéine Cactus séquestre un facteur de transcription Rel capable de transactiver ce gène codant pour un peptide antifongique. Finalement, en accord avec sa fonction inhibitrice, la protéine Cactus est effectivement exprimée dans le corps gras et est rapidement dégradée après infection microbienne [25]. En conclusion, ces données démontrent que la voie Toll/IL-1R est une cascade évolutivement ancienne impliquée dans les mécanismes de défense des insectes et des mammifères [26]. Très récemment, plusieurs gènes codant pour des protéines homologues du récepteur Toll ont pu être caractérisés chez l'homme. Ces gènes codent pour des protéines transmembranaires présentant un domaine extracytoplasmique riche en leucine, et un domaine cytoplasmique homologue du récepteur de l'interleukine-1 humaine comme la protéine Toll de drosophile [27]. Lorsque l'on transfecte une forme constitutivement active d'un de ces récepteurs dans des cellules de mammifères, on déclenche l'expression de gènes de cytokines de la réponse inflammatoire tels que $I L-1, I L-6$ et $I L-8$ [28]. Ainsi, les protéines Toll appartiennent à une famille de protéines conservées dans l'évolution et impliquées dans la réponse immunitaire. 


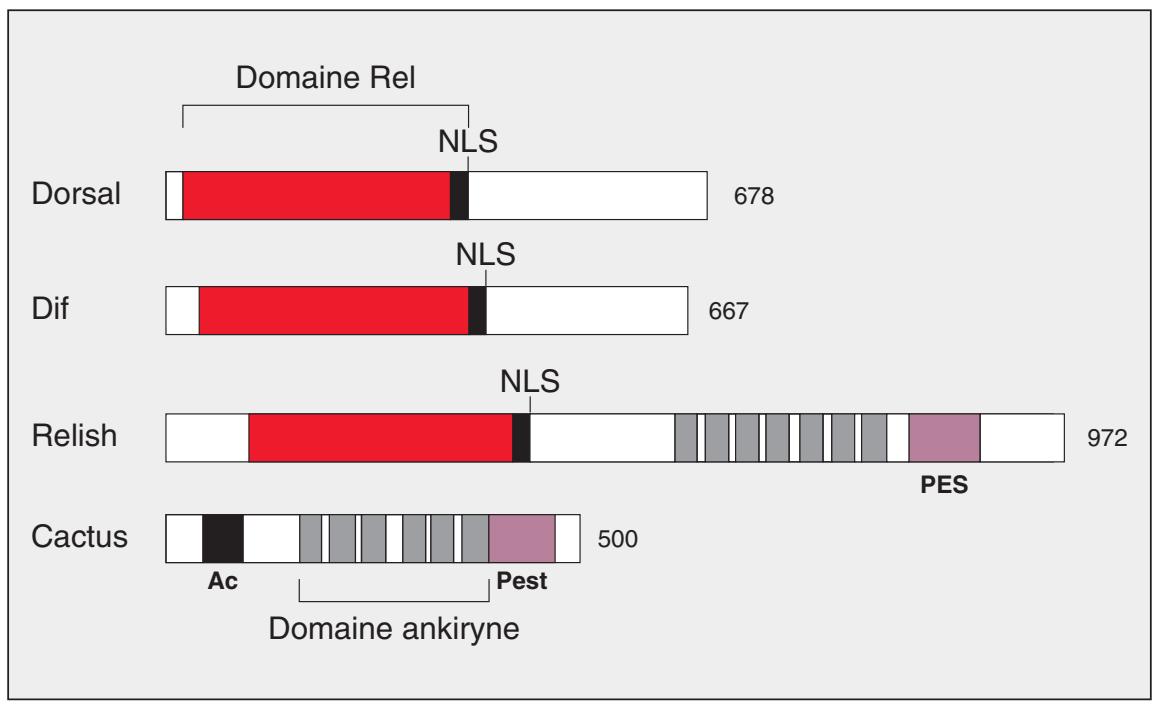

Figure 4. Les protéines Rel/NF-KB et IKB chez la drosophile. Les protéines de la famille Rel/NF- $K B$ possèdent dans leur région amino-terminale un domaine conservé d'environ 300 acides aminés appelé domaine Rel. Ce dernier comporte un domaine de liaison à I'ADN, un domaine de dimérisation et une séquence de localisation nucléaire (NLS). La région carboxy-terminale de Dorsal et Dif contient un domaine transactivateur. Les protéines I $B$ présentent plusieurs motifs de type ankyrine (domaine ankyrine) dans leur région carboxy-terminale. Cactus comporte un domaine très acide dans sa région amino-terminale (Ac). Relish et Cactus possèdent un domaine PEST généralement associé à un taux de dégradation élevé de la protéine. La structure de Relish est apparentée à celles de p105 et p100 qui sont les précurseurs respectifs des protéines Rel/NF-KB p50 et p52 chez les mammifères. Le nombre de résidus est indiqué à droite de chaque protéine. (D'après [4].)

On en serait peut-être resté là si des parallèles surprenants et quelque peu provocateurs n'avaient été établis entre des éléments de la voie Toll et des protéines de défense de plantes. En effet, plusieurs gènes de plantes impliqués dans la résistance à divers agents pathogènes codent pour des protéines présentant des similitudes avec des composants de la voie Toll/IL-1R [26]. Il en est ainsi des gènes $\mathrm{N}$ et $\mathrm{Pto}$ de tomate qui codent pour des protéines cytoplasmiques présentant des similitudes avec, respectivement, le récepteur Toll et la protéine-kinase Pelle. Enfin, un gène de résistance récemment caractérisé chez Arabidopsis thaliania, NPR1/NIM1, code pour une protéine qui partage des similitudes de séquence avec Cactus et IкB. On ne sait pas encore si ces protéines, qui ont été isolées chez des plantes d'espèces différentes, participent à une même voie de signalisation. Au vu de ces similitudes, certains biologistes pensent néanmoins que des voies de signalisation impliquées dans la défense
Un autre domaine d'intérêt concerne les protéines extracellulaires capables d'activer la voie Toll. Les données actuelles suggèrent que le ligand du récepteur Toll, Spätzle, est présent dans l'hémolymphe et serait activé par clivage protéolytique. Il est par conséquent crucial d'identifier les protéases impliquées dans la maturation de Spätzle. Les études génétiques indiquent que les protéases à sérine Easter, Snake, et Gastrulation Defective qui participent à la maturation de Spätzle dans l'embryon, ne sont pas nécessaires à l'activation de la voie Toll au cours de la réponse immunitaire [24]. Une hypothèse attrayante serait que des protéases impliquées dans les cascades protéolytiques comme la coagulation ou les réactions de mélanisation participent à la maturation de Spätzle.

\section{La réponse antibactérienne}

Des drosophiles portant des mutations activant la voie Toll expriment de façon constitutive le gène de la drosomycine. A l'inverse, aucune expression des gènes codant pour des peptides actifs uniquement contre les bactéries n'est observée chez ces mutants [24]. Cette observation indique qu'au moins deux voies de signalisation règlent la réponse antimicrobienne de la drosophile: la voie Toll qui contrôle la drosomycine et une autre cascade gouvernant l'expression des gènes codant pour les peptides antibactériens. Cette hypothèse a été confirmée par la caractérisation d'une nouvelle mutation, immune deficiency, qui bloque l'induction de tous les gènes codant pour les peptides antibactériens, sans altérer celle du gène de la drosomycine [29]. La nature moléculaire du gène immune deficiency n'est pas encore connue mais le clonage de ce gène sera une étape déterminante pour notre compréhension de la voie de signalisation activant la défense antibactérienne.

L'induction des gènes codant pour les peptides antibactériens cécropine, attacine et défensine, nécessite l'activation concomitante des voies Toll et immune deficiency (figure 5). A l'inverse, le gène de la metchnikowine peut être activé indifféremment par l'une ou l'autre des deux cascades [30]. 


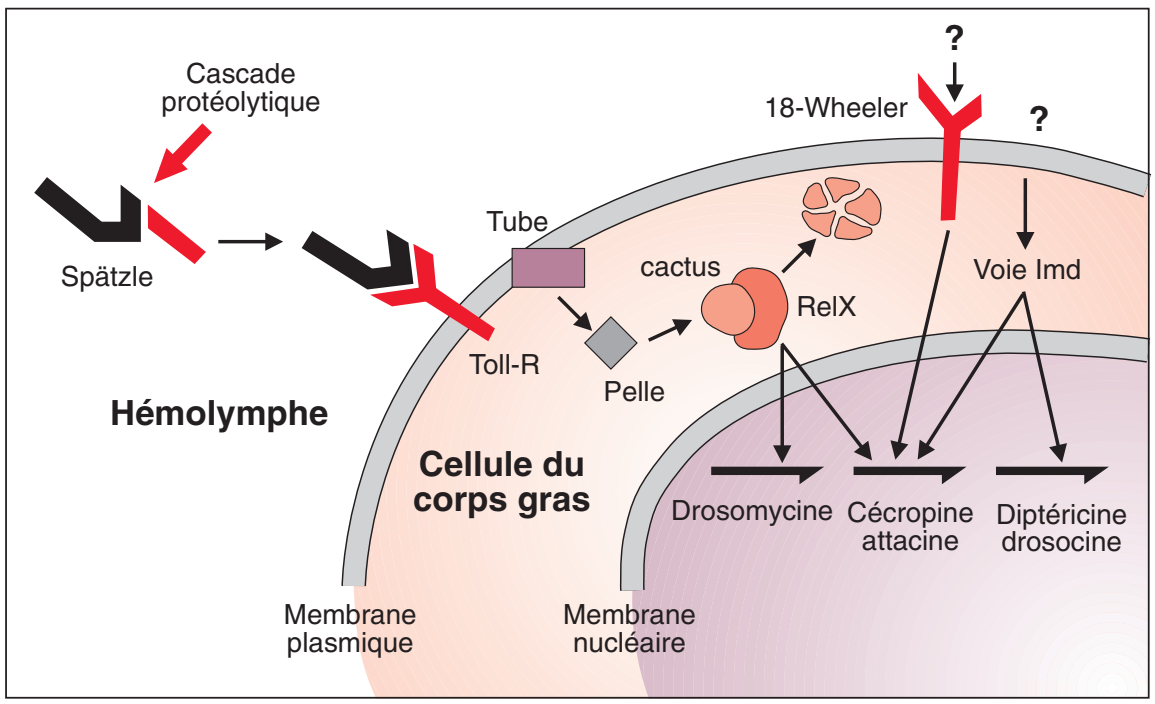

Figure 5. Modèle pour le contrôle de l'expression des gènes codant pour les peptides antimicrobiens dans le corps gras de drosophiles adultes. Selon ce modèle, une blessure septique activerait une cascade protéolytique aboutissant au clivage du précurseur de Spätzle. Spätzle activé se lierait alors au récepteur Toll. Cette fixation permettrait la transmission du signal intracellulaire qui, par l'intermédiaire des protéines Tube et Pelle, aboutirait à la dégradation de Cactus libérant la protéine Rel $X$ dans le noyau. Cette dernière irait activer le gène de la drosomycine et certains gènes codant pour des peptides antibactériens. La nature de la protéine Rel X n'est pas connue mais Dif seul (ou en synergie avec Dorsal) apparaît comme la meilleure candidate. La voie immune deficiency (Imd), qui règle l'expression de tous les gènes codant les peptides antibactériens, est encore inconnue. Finalement, le récepteur 18Wheeler, qui présente des similitudes avec Toll, règle par une cascade non caractérisée les gènes codant pour l'attacine et la cécropine. (D'après [4].)

Finalement, la voie Toll ne semble pas affecter l'expression des gènes codant pour la drosocine et la diptéricine (figure 5).

La caractérisation d'un nouveau gène, 18 wheeler, codant pour une protéine voisine du récepteur Toll et exprimé dans le corps gras, donne une image encore plus complexe de la réponse antimicrobienne [31]. En effet, le gène 18 wheeler est requis uniquement pour l'expression des gènes de l'attacine et de la cécropine mais pas pour celle des autres gènes codant pour des antimicrobiens. Il apparaît clairement que la réponse humorale antibactérienne met en jeu de multiples voies de signalisation qui sont résumées sur la figure 5. L'hypothèse actuelle est que chaque gène codant pour un peptide antimicrobien est contrôlé par un dosage particulier de ces différentes voies de signalisation. Ces cascades activent finalement des combinaisons différentes des protéines Rel Dorsal, Dif et Relish capables de se fixer sur les

gènes. Des cribles génétiques ayant pour but d'isoler de nouvelles mutations affectant la réponse humorale antimicrobienne ont été mis en œuvre dans différents laboratoires [32]. L'analyse moléculaire des gènes identifiés devrait permettre de compléter le schéma.

\section{Spécificité et reconnaissance}

La coexistence de multiples voies de signalisation gouvernant la réponse antimicrobienne conduit à se poser la question de la spécificité sousjacente à ces cascades. Des mécanismes spécifiques de reconnaissance d'une classe d'agent pathogène en amont de chacune de ces cascades permettraient une riposte adaptée à l'infection en ne produisant que les peptides actifs contre ces agents pathogènes. Plusieurs données récentes sont en faveur de cette hypothèse. Ainsi, il a été établi qu'effectivement la réponse antimicrobienne de la drosophile présente un certain niveau de spécificité [33]. Les gènes codant pour les peptides antimicrobiens sont induits de manière différente selon le type de micro-organisme injecté. Les gènes codant pour des peptides antibactériens comme la diptéricine, l'attacine, la drosocine, la défensine ou la cécropine sont très fortement induits après infection par des bactéries à Gram négatif. A l'inverse, les champignons et les bactéries à Gram positif sont les meilleurs inducteurs du gène codant pour la drosomycine. De plus, en utilisant des conditions naturelles d'infection, il a été démontré que la drosophile est bien capable d'induire une réponse adaptée [33]. En effet, le dépôt de spores d'un champignon entomopathogène, Beauveria bassiana, sur la cuticule de drosophile, induit une forte augmentation de l'expression du gène de la drosomycine, alors que les gènes codant pour les peptides antibactériens restent silencieux. Ce deutéromycète sécrète de nombreuses enzymes (protéases, chitinases, lipases) qui lui permettent de traverser la cuticule des insectes. Une étude génétique a permis de montrer que cette réponse adaptée est causée par une activation sélective de la voie Toll induite par la pénétration du champignon. Ces résultats démontrent que la drosophile est bien capable de différencier des classes d'agents pathogènes et donnent un sens biologique à l'existence de multiples voies de signalisation.

Les mécanismes de reconnaissance mis en jeu dans la réponse antimicrobienne ne sont pas connus. Ils pourraient faire intervenir des récepteurs capables de reconnaître des molécules aux motifs structuraux conservés, présents sur la paroi des procaryotes ou des champignons, mais absents des cellules hôtes. Charles Janeway a proposé, dès 1989, que de tels récepteurs soient appelés pattern recognition receptors et a suggéré qu'ils sont impliqués dans le déclenchement de la réponse innée [1]. Chez les mammifères, plusieurs pattern recognition receptors ont été identifiés: par exemple CD14, le récepteur des LPS [34], les collectines [35] et des récepteurs à large spectre de reconnaissance comme le scavenger receptor [36]. Ces récepteurs constituent une forme primitive de reconnaissance du nonsoi. Plusieurs travaux suggèrent l'exis- 
tence de tels récepteurs chez les insectes. Ainsi, une protéine du lépidoptère Bombyx mori, capable de se fixer aux parois des bactéries à Gram négatif a récemment été isolée et a été nommée GNBP (gram negative binding protein). Cette molécule est inductible après stimulation bactérienne et présente des similitudes de séquence partielles avec CD14 [37]. D'autres protéines présentant des similitudes structurales ou fonctionnelles avec des récepteurs de mammifères (scavenger receptor, CD36) ont été décrites chez la drosophile mais aucune preuve n'a été apportée quant au rôle de ces récepteurs dans le déclenchement de la réponse antimicrobienne de la drosophile [38, 39]. Finalement, d'autres mécanismes de reconnaissance du non-soi infectieux ne faisant pas intervenir de pattern recognition receptors existent probablement chez la drosophile. Par exemple, il n'est pas exclu que certains champignons entomopathogènes, tel Beauveria bassiana, puissent être reconnus non pas grâce à un déterminant particulier de l'enveloppe, mais par les protéases que ces agents pathogènes sécrètent afin de pénétrer la cuticule de l'insecte. De telles protéases pourraient, en clivant Spätzle ou une protéase de l'hôte, activer directement la voie Toll et induire une réponse antifongique.

\section{Fonction des peptides antimicrobiens}

Des peptides antimicrobiens ont été trouvés chez des organismes aussi variés que les insectes, les plantes et les mammifères. Il est généralement admis qu'ils jouent un rôle important dans la défense contre les infections. La caractérisation des mutations immune deficiency et Toll qui réduisent considérablement la synthèse des peptides antimicrobiens a permis d'aborder la fonction in vivo de ces peptides dans la lutte contre les infections [24, 29]. Des expériences ont montré que les mutants immune deficiency, qui ne produisent plus de peptides antibactériens, présentent une forte susceptibilité aux infections par la bactérie Escherichia coli par comparaison avec des drosophiles sauvages ou mutées pour Toll. Chez ces mêmes mutants, on observe une augmentation d'un facteur mille du nombre de bactéries 24 heures après infection alors qu'aucune croissance bactérienne n'est observée chez des drosophiles sauvages ou déficientes pour le récepteur Toll. Réciproquement, les mutants déficients pour la voie Toll, qui ne produisent plus de drosomycine, présentent une forte susceptibilité aux infections par le champignon Aspergillus fumigatus par comparaison avec des drosophiles sauvages ou mutées pour immune deficiency. Ainsi, la perte d'inductibilité d'une classe de peptides (antibactériens/antifongique) est corrélée à une susceptibilité accrue aux infections par les micro-organismes contre lesquels les peptides sont dirigés. Bien que l'on ne puisse pas exclure que les mutations testées affectent d'autres processus essentiels pour la survie de la drosophile, ces données sont en faveur d'un rôle important des peptides antimicrobiens dans la défense de la drosophile [24].

\section{Conclusions et perpectives}

Il y a quelques années, certains biologistes auraient pu être choqués d'entendre parler d'immunologie pour un organisme qui, comme la drosophile, est dépourvu d'anticorps, de lymphocytes, de mémoire immunitaire... enfin de ce qui fait l'essentiel des ouvrages d'immunologie. Le fait que les insectes sont, malgré l'absence de tout l'arsenal du système immunitaire adaptatif, très résistants aux infections apparaissait quelque peu paradoxal. La redécouverte du rôle fondamental de la réponse immunitaire innée chez les mammifères a profondément bouleversé notre perception des mécanismes de défense et donne une portée plus générale aux travaux réalisés chez l'insecte. La réponse innée, qui effectue depuis des centaines de millions d'années de manière très efficace la défense des invertébrés contre les infections microbiennes, a été conservée chez les vertébrés. Cette prise de conscience permet de mieux comprendre l'origine du système adaptatif qui a été construit sur une base évolutive plus ancienne et conservée phylogénétiquement. Ainsi, insectes et mammifères présentent de nombreuses réactions de défense semblables: réaction de coagulation, pha- gocytose, synthèse des peptides antimicrobiens. Il est probable que, comme c'est le cas pour la voie Toll, les bases moléculaires de ces mécanismes soient aussi conservées. Ainsi, à l'instar de ce qu'elle a représenté pour la compréhension du développement, la drosophile apparaît comme un modèle prometteur pour dévoiler certains des mécanismes qui règlent la réponse immunitaire innée chez l'homme. La caractérisation, à la suite des travaux chez la mouche, d'un homologue humain du récepteur Toll qui est aussi impliqué dans les réactions immunitaires, en est une illustration récente [28]. Outre sa génétique très performante, le modèle drosophile permet d'étudier les mécanismes de défense innée sans qu'il y ait interférence avec la réponse adaptative comme c'est le cas chez les mammifères chez lesquels les deux réponses sont étroitement intriquées. L'étude des réactions immunitaires de la drosophile présente d'autres intérêts qui tiennent au rôle important des insectes tant au plan économique que sanitaire. Ainsi ces études permettent de concevoir de nouvelles approches pour prévenir les infections chez les insectes utiles (vers à soie, abeilles), ou au contraire lutter contre les insectes nuisibles. Par exemple, les informations acquises chez la drosophile peuvent aider à mieux comprendre comment Plasmodium falciparum, le parasite à l'origine du paludisme, peut déjouer les défenses immunitaires de son hôte Anopheles gambiae pour concevoir des souches de moustiques résistantes au parasite [40].

Finalement, les résultats obtenus chez la drosophile indiquent des similitudes frappantes entre les mécanismes de défense antimicrobienne des animaux et ceux des plantes. Ils soulignent ainsi l'universalité et l'origine évolutive très ancienne de ces réactions immunitaires

\section{Remerciements}

Je remercie chaleureusement tous mes anciens collègues de l'UPR 9022 (Cnrs, Strasbourg), en particulier Jules Hoffmann, directeur du laboratoire et Marie Meister pour leur soutien et leurs commentaires sur le manuscrit. Je remercie également les Drs Serge Ohresser et Dominique Ferrandon pour l'aide apportée à l'illustration de cette revue. 


\section{RÉFÉRENCES}

1. Medzhitov R, Janeway JCA. Innate immunity: the virtues of a nonclonal system of recognition. Cell 1997; 91 : 295-8.

2. Fearon DT, Locksley RM. The instructive role of innate immunity in the acquired immune response. Science 1996; 272: 50-4.

3. Meister M, Lemaître B, Hoffmann JA. Antimicrobial defense in Drosophila. BioEssay $1997 ; 19: 1019-26$.

4. Hoffmann JA, Reichhart JM, Hetru C. Innate immunity in higher insects. Curr Opin Immunol 1996; 8: 8-13.

5. Ferrandon D, Jung A, Criqui MC, et al. A drosomycin-GFP reporter transgene reveals a local immune response in Drosophila that is not dependent on the Toll pathway. EMBO J 1998; 17 : 1217-27.

6. Ganz T, Lehrer R. Antimicrobial peptides of vertebrates. Curr Opin Immunol 1998; 10 : 41-4.

7. Georgel P, Meister M, Kappler C, Lemaitre B, Reichhart JM, Hoffmann JA. Insect immunity: the diptericin promoter contains multiple functional regulatory sequences homologous to mammalian acute-phase response elements. Biochem Biophys Res Commun 1994; 197 : 508-17.

8. Meister M, Braun A, Kappler C, Reichhart JM, Hoffmann JA. Insect immunity. A transgenic analysis in Drosophila defines several functional domains in the diptericin promoter. EMBO J 1994; 13: 5958-66.

9. Kadalayil L, Petersen UM, Engström Y. Adjacent GATA and kB-like motifs regulate the expression of a Drosophila immune gene. Nucleic Acids Res 1997; 25 : 1233-9.

10. Engström Y, Kadalayil L, Sun SC, Samakovlis C, Hultmark D, Faye I. kB-like motifs regulate the induction of immune genes in Drosophila. J Mol Biol 1993; 232: 327-33.

11. Kappler C, Meister M, Lagueux M, Gateff E, Hoffmann JA, Reichhart JM. Insect Immunity. Two 17 bp repeats nesting a kB-related sequence confer inducibility to the diptericin gene and bind a polypeptide in bacteria-challenged Drosophila. EMBO J $1993 ; 12$ : 1561-8.

12. Costello R, Lecine P, Kahn-Perlès B, et $a l$. Activation du système de transcription Rel/NF-kB. Med Sci 1995; 11 : 957-65.

13. Israël A. Les protéines Rel/NF-kB et IkB : nouvelles données sur la structure, la fonction et la régulation. Med Sci 1995; 11 : 1017-20.

14. Stancovski I, Baltimore D. NF-kB activation: the IkB kinase revealed? Cell 1997; 91: 299-302.

15. Steward R. Dorsal, an embryonic polarity gene in Drosophila is homologous to the vertebrate proto-oncogene, c-rel. Science 1987; 238: 692-4

16. Ip TY, Reach M, Engström Y, et al. Dif, a dorsal-related gene that mediates an immune response in Drosophila. Cell 1993; $75: 753-63$.

17. Dushay M, Asling B, Hultmark D. Origins of immunity: relish, a compound Rel-like gene in the antibacterial defense of Drosophila.
18. Reichhart JM, Georgel P, Meister M, Lemaitre B, Kappler C, Hoffmann JA. Expression and nuclear translocation of the rel NF-kB-related morphogen dorsal during the immune response of Drosophila. $C R$ Acad Sci Paris Life Sci 1993; 316: 1207-17.

19. Lemaître B, Meister M, Govind S, et al. Functional analysis and regulation of nuclear import of dorsal during the immune response in Drosophila. EMBO J 1995; 14 : 536-45.

20. Petersen UM, Björklund G, Ip YT, Engström Y. The dorsal-related immunity factor Dif, is a sequence-specific trans-activator of Drosophila cecropin gene expression. EMBO $J 1995$; 14 : 3146-58

21. Gross I, Georgel P, Kappler C, Reichhart JM, Hoffmann JA. Drosophila immunity: comparative analysis of the Rel proteins dorsal and Dif in the induction of the genes encoding diptericin and cecropin. Nucleic Acids Res 1996; 24 : 1238-45.

22. Salier JP, Rouet P, Banine F, Claeyssens $S$. Transcription des gènes de protéines plasmatiques dans le foie au cours de l'inflammation aiguë systémique. Med Sci 1997; $13: 335-44$.

23. Belvin MP, Anderson KV. A conserved signaling pathway: the Drosophila Toll-Dorsal pathway. Annu Rev Cell Dev Biol 1996; 12 393-416.

24. Lemaitre B, Nicolas E, Michaut L, Reichhart JM, Hoffmann JA. The dorsoventral regulatory gene cassette spaetzle/Toll/cactus controls the potent antifungal response in Drosophila adults. Cell 1996; 86: 973-83.

25. Nicolas E, Reichhart JM, Hoffmann JA Lemaitre B. In vivo regulation of the I I B homologue cactus during the immune response of Drosophila. J Biol Chem 1998; 273: 10463-9.

26. Medzhitov R, Janeway Jr CA. An ancient system of host defense. Curr Opin Immunol $1998 ; 10: 12-5$

27. Rock FL, Hardiman G, Timans JC, Kastelein RA, Bazan JF. A family of human receptors structurally related to Drosophila Toll. Proc Natl Acad Sci USA 1995; 95 : 588-93.

28. Medzhitov R, Preston-Hurlburt P, Janeway CA. A human homologue of the Drosophila Toll protein signals activation of adaptive immunity. Nature 1997; 388: 394-7.

29. Lemaitre B, Kromer-Metzger E, Michaut $\mathrm{L}$, et al. A recessive mutation, immune defi ciency (imd), defines two distinct control pathways in the Drosophila host defense. Proc Natl Acad Sci USA 1995; 92 : 9465-9.

30. Levashina EA, Ohresser S, Lemaitre B, Imler JL. Two distincts pathways can control expression of the gene encoding the Drosophila antimicrobial peptide metchnikowin. $J$ Mol Biol 1998; 278: 515-27.

31. Williams MJ, Rodriguez A, Kimbrell DA Eldon ED. The 18-wheeler mutation reveals complex antibaterial gene regulation in Drosophila host defense. EMBO J1997; 16: 6120-30.

32. Wu LP, Anderson KV. Regulated nuclear import of Rel proteins in the Drosophila immune response. Nature 1998; 392: 93-7.

33. Lemaître B, Reichhart JM, Hoffmann JA. Drosophila host defense: differential induction of antimicrobial peptide genes after infection by various classes of microorganisms. Proc Natl Acad Sci USA 1997; 94 . 14614-9.

34. Ulevitch RJ, Tobias PS. Receptor-dependent mechanisms of cell stimulation by bacterial endotoxin. Ann Rev Immunol 1995 $13: 437-57$.

35. Epstein J, Eichbaum Q, Sheriff S, Ezekowitz $\mathrm{RAB}$. The collectins in innate imunity. Curr Opin Immunol 1996; 8 : 29-35.

36. Pearson A. Scavenger receptor in immunity. Curr Opin Immunol 1996; 8: 20-8

37. Lee WJ, Lee JD, Kravchenko VV, Ulevitch RJ, Brey PT. Purification and molecular cloning of an inducible Gram-negativebacteria-binding protein from silkworm, Bombyx mori. Proc Natl Acad Sci USA 1996 93: 7888-93.

38. Franc N, Dimarcq JL, Lagueux M, Hoffmann JA, Ezekowitz RAB. Croquemort, a novel Drosophila hemocyte/macrophage receptor that recognizes apoptotic cells Immunity 1996 ; 4 : 431-43

39. Pearson A, Lux A, Krieger M. Expression cloning of dSR-CI, a class C macrophage-specific scavenger receptor from Drosophila melanogaster. Proc Natl Acad Sci USA $1995 ; 92$ : 4056-60

40. Richman A, Kafatos FC. Immunity to eukaryotic parasites in vector insects. Curr Opin Immunol 1997; 8 : 14-9.

\section{Summary}

Drosophila: a model

for the understanding

of innate immunity

Drosophila as other insects are particularly resistant to microbial infection. Drosophila host defense system relies on several innate reactions: immediate activation of two proteolytic cascades leading to blood clotting and to melanization, phagocytosis and encapsulation of intruders by bloods cells, induced synthesis by the fat body of a battery of antimicrobial peptides which are secreted into the hemolymph. Recent genetic analysis has revealed striking similarities between signaling pathways leading to antimicrobial peptide gene expression in Drosophila and cytokine-induced expression of the genes coding for acute phase proteins in mammals. The finding that insect and mammalian immunity involve related signaling pathways offers the promise that genetic studies in Drosophila might contribute to our understanding of the innate immune response in higher organisms. 